Physical Sciences | John Lehman \& Paul Williams

\section{Weighing weightless photons for laser-based manufacturing}

It may be hard to believe
that a beam of light can melt
steel, but that is exactly what
happens during laser beam
machining. Using lasers for
processing has become
increasingly common but
knowing exactly how much
energy and heating occurs
during these processes is a
challenging task. This is why
colleagues Dr John Lehman and
Dr Paul Williams at the National
Institute of Standards and
Technology (NIST) in Boulder,
USA, have been finding ways
to use photon momentum to
measure the force of light -
research in this area, along with
the redefinition of the kilogram,
changes how we think of the
optical Watt.

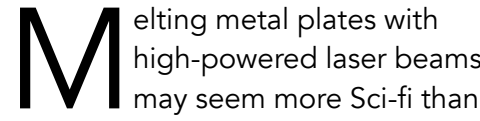
mundane industrial processing. Still, the advent of more robust high-power lasers systems has meant that laser beam machining has become a routin part of many industries. Laser beams make excellent tools as they do not wear with time, are energy efficient,

Laser-based manufacturing often involves taking a high-power laser of a metal. The beam contains many packets of light energy known as photons, and when the metal absorbs these photons, a tremendous amount of energy is transferred and converted to heat which melts the metal, thus welding or cutting it depending on the processing conditions. An advantage of this laser-based approach is that the heating process is very localised, meaning the surrounding material is less likely to deform or lose its strength.

The high levels of precision offered by high-power laser machining have even led to new $3 \mathrm{D}$ printing techniques for fusion, which laser powdercreate high performing und compex structures To maximise the bempeits laser machining means having not just excellent control over the position and movement of the laser beam, but also of the energy it imparts. It is therefore necessary to accurately measure the power of the laser beam in real time during its operation.

However, instruments capable of measuring the power of high-power beams to very high accuracies tend to

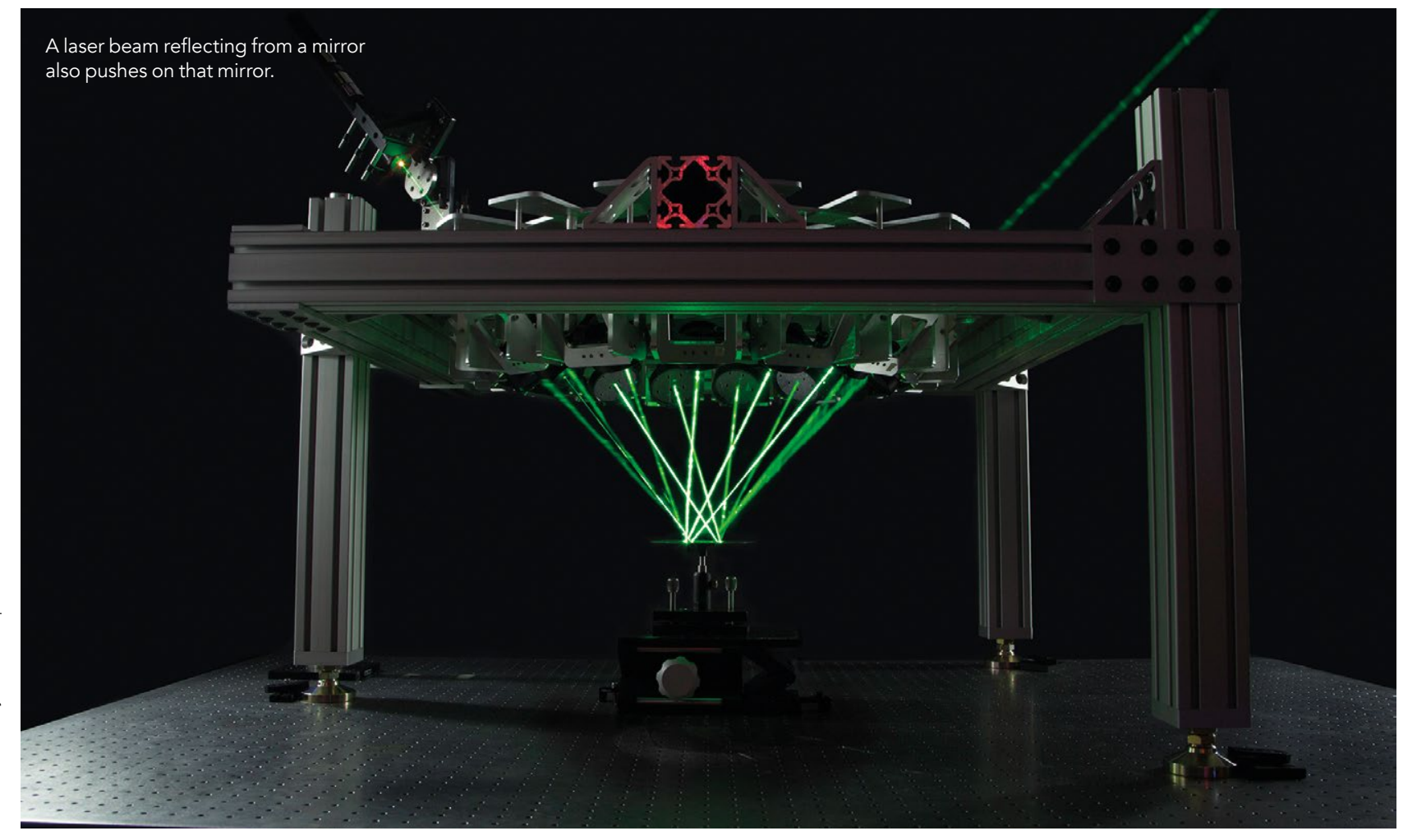

be large and impractical to integrate with machining processes. Normally, laser power by measuring a change in emperature of the detector caused by the absorbed photons. Dr John Lehman, Dr Paul Williams and their colleagues at the National Institute of Standards and Technology (NIST) in the US have taken a different approach to measure optical power.

Rather than measuring thermal changes, the research team has been exploiting the concept of photon momentum. When a tennis ball speeds towards a stationary racket and bounces off, as it bounces it will transfer momentum to the racket, causing the racket to move. It turns thing phon you shine a mirror. By measuring the push (force) of a laser beam as it reflects from a mirror the power in that laser beam is also determined.

\section{THE WEIGHTLESS SCALE}

A mechanical kitchen scale measures weight by stretching a spring that is attached to a moving dial - which is what you read on the outside of the scale to see the weight. The heavier the object, the greater the force to stretch the spring and the further the dial moves. Similarly, the higher the power of a laser beam, the higher the numb of photons that will be travelling in the beam - thus pushing harder on
the mirror and making the 'dial' move further. The force of the reflal moved photons on the mirror is known as photons on the mirror
'radiation pressure'.

Unlike flour and most things in the kitchen, photons are massless objects, so 'weighing' the photons in this way is really measuring their momentum. A laser beam of 100 kilowatts contains nearly a trillion-trillion photons but ends up 'weighing' the same amount as two paper staples. Dr Lehman and DrWilliams have found the ability to measure photon momentum to be a powerful tool for precisely measuring laser powers.

By assessing the photon's momentum as it reflects from a mirror, a non-

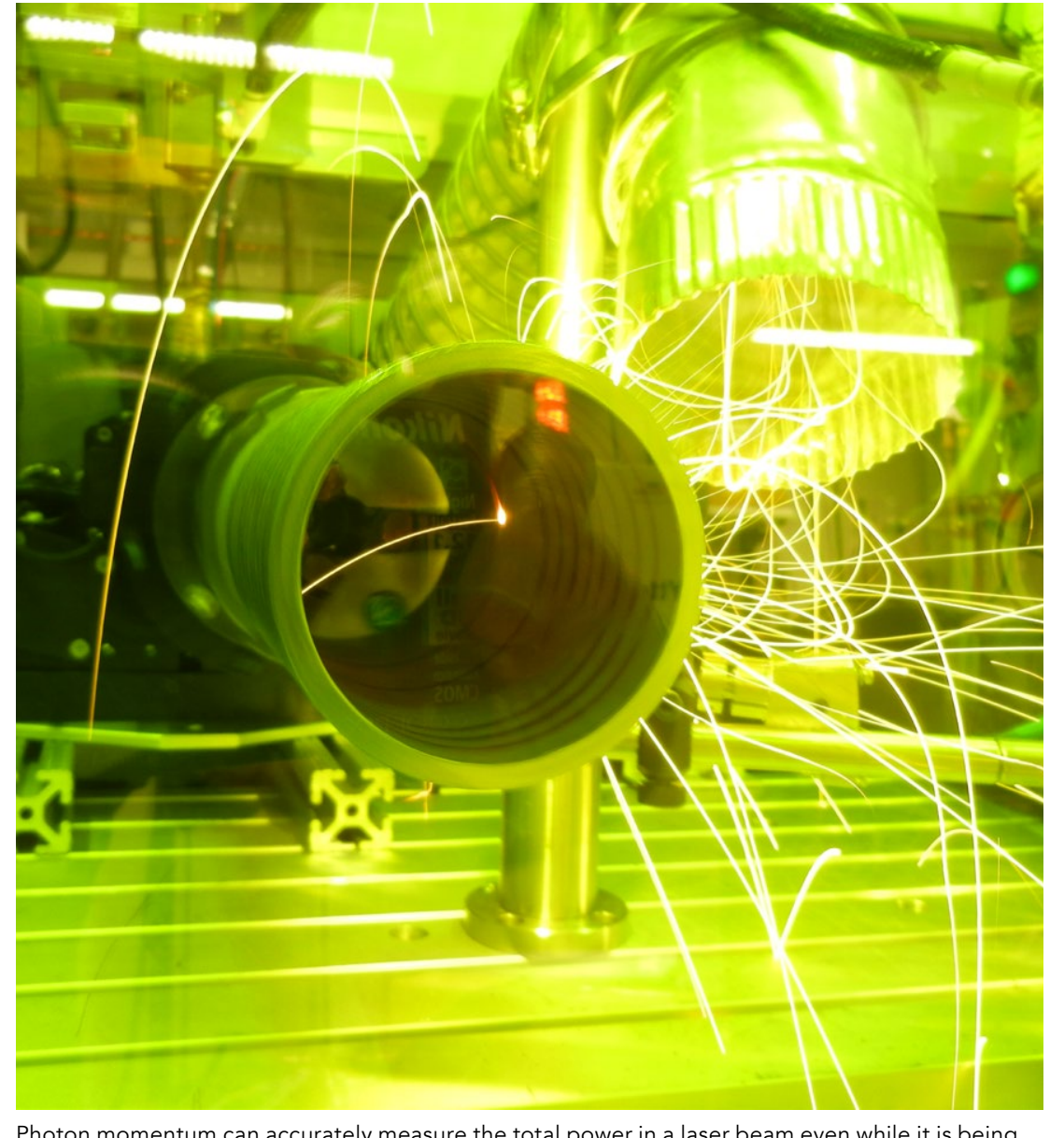

Photon momen
used to weld.

laser power can be made without interfering with the experiment. The method works over a range of laser powers, not just the very intense beams used in manufacturing, and is also non-destructive, with great promer monitoring te, hiq laser

performed their research, are very concerned with finding ways to perform and standardise scientific measurements so they are both accurate and repeatable. This is crucially important work as scientific instruments for precision measurements

Dr Lehman and Dr Williams have found the ability to measure photon momentum to be a powerful tool for precisely measuring laser powers.

REDEFINING THE KILOGRAM Radiation pressure is not only enabling laser power measurement, but is even related to the changing of the definition of a unit many of us will use in our dayto-day lives: the kilogram

Metrology is often called the science of measurement, and institutes Dr Williams and their colleagues relative changes (i.e. whether the by 2 degrees) and absolute ones (i.e. whether the temperature is 20 degrees
or 25 degrees).

Standards and references have an important role to play in science. System of Units (SI) wa In denational so that when someone claimed they 


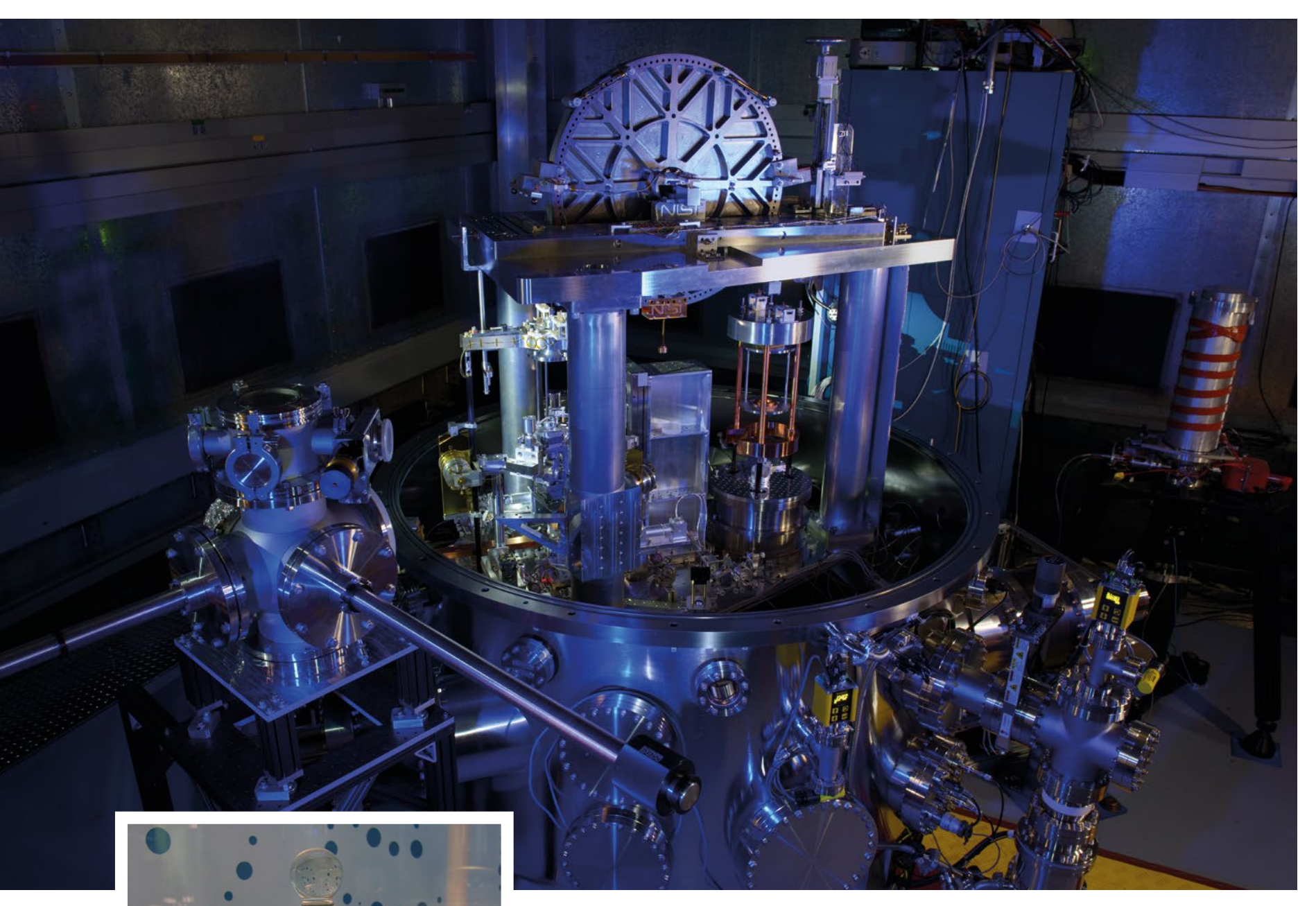

In 2018 though, something unusual happened. The definition of a kilogram changed.

changed. Rather than relying on a momentum is so important, and why platinum alloy cylinder remaining $\quad$ Dres Lehman and Willams are exploring corrosion, the International Bureau of radiofrequency and microwave power Weights and Measures voted to instead with an analytical balance. rely on Planck's constant. Planck's constant is considered to be one of the laser power measuring devices that $\begin{array}{ll}\text { in a Kibble balance, or by measuring } & \text { can operate in even very extreme } \\ \text { conditions where thermal power }\end{array}$ the photon momentum using a mirror, meters fail, the researchers hope that just as Drs Lehman and Williams have one day that radiation pressure power done to look at laser powers. Their metres will be appearing in textbooks approach is an elegant way of making alongside thermal and quantum such measurements. detectors. For laser welding, this could OUTLOOK

For metrologists, being able to make measurements that are calibrated to a low uncertainty is of utmost for measuring laser pow aproaches capable of fully characterising, and in manufacturing with the ability

\section{Behind the Research}

Dr John

Lehman

DrPaul

Williams

Research Objectives

Dr Lehman and Dr Williams establish a novel measurement scheme, photon momentum, as a means to measure laser power.

Detail

325 Broadway

Boulder

CO, 80305
USA

Bio

John Lehman is leader of the Sources and Detectors Group at the Nationa

(NIST). He is a Fellow of the Alexander

von Humboldt Eoundation of Germary

and the Optical Society (OSA). John was awarded the Quantum Electronics and Photonics PhD prize from the Institute of Physics (UK) and other awards.

\section{References}

Williams, P., Hadler, J., Maring, F., Lee, R., Rogers, K.

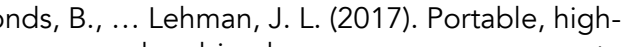
accuracy, non-absorbing laser power measurement at
kilowatt levels by means of radiation pressure. Optics Express, 25(4), 4382-4392.

Artusio-Glimpse, A. B., Ryger, I., Azarova, N. A., Williams, P. A., Hadler, J. A., \& Lehman, J. H. (2020). Miniature force sensor for absolute laser power measurements vi radiation pressure at hund

Williams, P. A., Spidell, M. T., Hadler, J. A., Gerrits, T., study of laser power calibrations magnitude with traceability to the kilogram. Metrologia, 57,015001

Lehman, J. H. Rogers, K., Rahn, D. \& Williams, P. (2019). . mean radiation pressure monitors therefore controlling, the process leading to better quality control and structures. 